\title{
Selumetinib Inhibits Melanoma Metastasis to Mouse Liver via Suppression of EMT-targeted Genes
}

\author{
SEUNG-HEE RYU ${ }^{1}$, SEUNG-HO HEO ${ }^{2}$, EUN YOUNG PARK ${ }^{1}, \mathrm{KYUNG}^{-C H U L} \mathrm{CHOI}^{3}$, \\ JE-WON RYU ${ }^{2}$, SEOK HO LEE ${ }^{4}$ and SANG-WOOK LEE ${ }^{1}$ \\ Departments of ${ }^{1}$ Radiation Oncology and ${ }^{3}$ Biomedical Science, Asan Medical Center, \\ University of Ulsan College of Medicine, Seoul, Republic of Korea; \\ ${ }^{2}$ Asan Institute for Life Sciences, Asan Medical Center, Seoul, Republic of Korea; \\ ${ }^{4}$ Department of Radiation Oncology, Gachon University Gil Medical Center, Incheon, Republic of Korea
}

\begin{abstract}
Aim: We investigated the therapeutic effects of a mitogen-activated protein (MEK) inhibitor, selumetinib, in a hepatic melanoma metastasis model and studied its possible mechanism of action. Materials and Methods: Melanoma cell lines were exposed to selumetinib under different experimental conditions. We established a mouse model of liver metastasis and treated mice orally with vehicle or selumetinib and then evaluated metastasis progress. Results: Growth inhibition was observed in melanoma cells as a consequence of $G_{1}$-phase cell-cycle arrest and the subsequent induction of apoptosis in a doseand time-dependent manner. Mice with established liver metastases that were treated with selumetinib exhibited significantly less tumor progression than vehicle-treated mice. c-Myc expression in metastasized liver tissues were suppressed by selumetinib. Moreover, oral treatment with selumetinib modulated expression of epithelial-tomesenchymal transition- and metastasis-related genes, including integrin alpha-5 (ITGA5), jagged 1 (JAG1), zinc finger E-box-binding homeobox 1 (ZEB1), NOTCH, and serpin peptidase inhibitor clade E (SERPINE1). Conclusion: We established a mouse model of hepatic metastasis using a human melanoma cell line, such models are essential in elucidating the therapeutic effects of anti-metastatic drugs. Our data suggest the possibility that selumetinib presents a new strategy to treat liver metastasis in patients with melanoma by suppressing epithelial-to-mesenchymal transition-related genes.
\end{abstract}

Correspondence to: Professor Sang-wook Lee, Department of Radiation Oncology, University of Ulsan College of Medicine, Asan Medical Center, 88, Olympic-ro 43-gil, Songpa-gu, Seoul, 05505, Republic of Korea. Tel: +82 230104435, e-mail: 1sw@amc.seoul.kr

Key Words: MEK inhibitor, selumetinib (AZD6244), liver metastasis, melanoma, EMT.
Malignant melanoma is one of the most fatal diseases and exhibits a very high metastatic potential (1). Moreover, patients with metastatic melanoma have extremely poor survival outcomes (2). Despite successful control of the primary tumor, metastatic disease ultimately develops in $\sim 35 \%$ of affected patients. Common sites of metastasis from melanoma include the lung, liver, brain and bones, with the liver being the most common site of metastases (3). Liver metastases are generally refractory to systemic chemotherapy, and the median survival for patients with liver metastases is $\sim 6$ months. Irrespective of treatment, the mortality rate is $\sim 90 \%$ at 2 years, with only $\sim 1 \%$ of patients surviving more than 5 years (4). Liver resection represents an option for patients with few liver metastases (5). However, few therapeutic options exist for patients with more extensive tumor burdens.

The poor prognosis of melanoma necessitates the development of novel therapeutic agents to improve the overall survival rate of affected patients. Furthermore, treatment for advanced melanoma is extremely challenging, as only two drugs, dacabazine and recombinant human highdose interleukin 2, have been approved for the treatment of metastatic melanoma by the US Food and Drug Administration (5). Because of the limited therapeutic options, novel drug targets are urgently needed to improve prognosis for this most-aggressive lethal cancer.

$B R A F$ mutations have been identified in more than $70 \%$ of malignant melanomas. Active BRAF signals through mitogen-activated protein (MEK) to activate aberrant mitogen-activated protein kinase (MAPK)/extracellular signal-regulated kinase (ERK) pathway, which in turn activates downstream transcription factors (6). In most melanomas, the ERK pathway is constitutively active and plays a role in many oncogenic processes $(7,8)$. Thus, blockade of MEK/ERK signaling may represent an effective approach for anticancer therapy. A RAF inhibitor (sorafenib) has been tested in the clinic as a targeted therapeutic approach $(9,10)$; however, its efficacy was found to be 
Table I. Primer sequences used in the study.

\begin{tabular}{lll}
\hline Target gene & Name & Primer sequences \\
\hline COL1A2 & Collagen type I alpha 2 chain & F: CCTGGTAATCCTGGAGCAAA \\
R: CAGATCCAGCTTCCCCATTA & F: CGACGCTCTTTACCGTTCTC \\
R: GCCATGCCGAAGAAGTAGAG & F: CACTGGCCATGATGAGTTTG \\
ITGA5 & Frizzled-7 & R: GGATATCCATTGCCATCCAG \\
JAG1 & Integrin alpha-5 & F: AAGGAAAGACCTGCCACTCA \\
& & R: GGGCTGCAGTCATTGGTATT \\
MMP9 & Jagged 1 & F: CATCGTCATCCAGTTTGGTG \\
& & R: TCGAAGATGAAGGGGAAGTG \\
Notch & Matrix metallopeptidase 9 & F: GTCAACGCCGTAGATGACCT \\
& & R: CAGGTTGTACTCGTCCAGCA \\
SERPINE1 & NOTCH & F: CTCTCTCTGCCCTCACCAAC \\
& & R: GTGGAGAGGCTCTTGGTCTG \\
TGF 31 & Serpin peptidase inhibitor, clade E & F: AAGTGGACATCAACGGGTTC \\
& & R: TGCGGAAGTCAATGTACAGC \\
ZEB1 & Transforming growth factor beta 1 & F: TGCACTGAGTGTGGAAAAGC \\
GAPDH & & R: TGGTGATGCTGAAAGAGACG \\
& Zinc finger E-box-binding homeobox 1 & F: GACCCCTTCATTGACCTC \\
\hline
\end{tabular}

insufficient. Therefore, alternative aspects of BRAF signaling, such as MEK, had to be considered. Many MEK inhibitors are currently undergoing preclinical and early clinical evaluation for various cancer types, including melanoma (11-14). Several preclinical and clinical studies have indicated that the MEK inhibitor, selumetinib (AZD6244), which is a potent and selective inhibitor of MEK1 and MEK2, exhibits promising antitumor activity (7, 13). However, a phase II study reported disappointing results because of either suboptimal bioavailability or insufficient inhibition of the targeted signaling pathway (15). Moreover, it is unknown whether a MEK inhibitor can be effective against melanoma metastasis, especially in the liver. In this study, we investigated the effect of selumetinib on hepatic metastasis and elucidated the related mechanisms.

\section{Materials and Methods}

Cell culture and reagents. Melanoma cell lines M14 and UACC62 were purchased from the American Type Culture Collection (Manassas, VA, USA). Cells were cultured in RPMI-1640 medium supplemented with $10 \%$ fetal bovine serum (FBS), $50 \mu \mathrm{g} / \mathrm{ml}$ penicillin, and $100 \mu \mathrm{g} / \mathrm{ml}$ streptomycin in a humidified $37^{\circ} \mathrm{C}$ incubator with $5 \%$ $\mathrm{CO}_{2}$. The MEK inhibitor, selumetinib (AZD6244, ARRY-142886) was purchased from Selleck Chemicals (Houston, TX, USA). AZD6244 was dissolved in dimethyl sulfoxide and then diluted in fresh medium to adequate concentrations for in vitro experiments.

Western blot analysis. Melanoma cells were cultured in complete medium to $\sim 70 \%$ confluence and then different concentrations $\left(10^{-3}-10 \mu \mathrm{M}\right)$ of selumetinib were added for 24 or $48 \mathrm{~h}$ as appropriate. Cell lysates were prepared in cell lysis buffer (Cell Signaling Technology, Beverly, MA, USA) containing protease and phosphatase inhibitor cocktails. Proteins were subjected to sodium dodecyl sulfate -polyacrylamide gel electrophoresis (SDS-PAGE) and transferred to a polyvinylidene difluoride membrane (Amersham Biosciences, Freiburg, Germany). Blots were probed with primary antibodies that recognized the following antigens: phospho-ERK (Cell Signaling), ERK (Cell Signaling), $\beta$-actin (Sigma, St. Louis, MO, USA), c-MYC (Cell Signaling), cyclin D1 (Cell Signaling), poly ADP ribose polymerase (PARP) (Cell Signaling), p27 (Cell Signaling), and survivin (Abcam, Cambridge, UK). Proteins were visualized using an enhanced chemiluminescence system (Amersham Biosciences, Freiburg, Germany). Protein expression levels in liver tissues were also assessed by western blot analysis. Tissue lysates were prepared in cell lysis buffer (Intron, Seongnam, Korea) and other procedures were carried out as described above.

Cell-cycle distribution and annexin $V$ staining. Cells were treated with selumetinib for 24,48 , or $72 \mathrm{~h}$ and then stained using propidium iodide (PI) in appropriate buffer. DNA content was determined using a FACScan flow cytometer (Becton Dickinson, Franklin Lakes, NJ, USA) equipped with a $488 \mathrm{~nm}$ argon laser. The percentages of cells in subG1, $G_{0} / G_{1}, S$, and $G_{2}$ phases were analyzed using ModFit LT software (Becton Dickinson, San Diego, CA, USA). To analyze apoptosis, cells were treated with selumetinib, stained with an Annexin V Apoptosis Detection Kit (BD Biosciences Pharmingen, San Diego, CA, USA), and analyzed using a flow cytometer.

RNA extraction and gene-expression analysis. Total RNA was isolated using the RNeasy Kit (Qiagen, Hilden, Germany) and subjected to reverse transcription using the PrimeScript ${ }^{\mathrm{TM}}$ reverse transcriptase kit (TaKaRa Bio Inc., Otsu, Japan) to generate first-strand cDNA. 
A

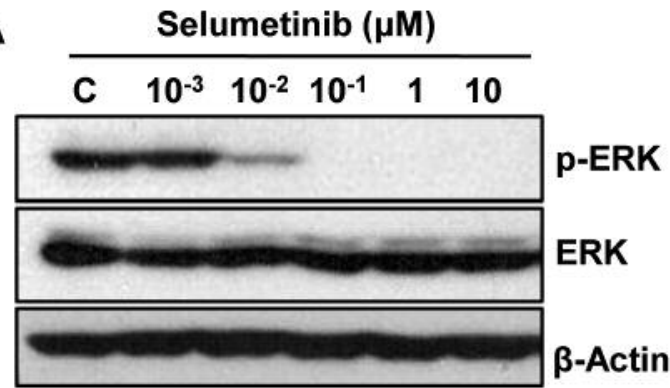



Figure 1. Effects of mitogen-activated protein (MEK) inhibitor on constitutive extracellular signal-regulated kinase (ERK) phosphorylation in human melanoma cell lines M14 (A) and UACC62 (B). Cells were plated in 6-well plates and exposed to MEK inhibitor (selumetinib) at the indicated concentrations for $24 \mathrm{~h}$.

Polymerase chain reaction (PCR) was then carried out for 30-35 cycles (depending on the primers) with the following cycling conditions: $95^{\circ} \mathrm{C}$ for $1 \mathrm{~min}, 55^{\circ} \mathrm{C}$ for $1 \mathrm{~min}$, and $72^{\circ} \mathrm{C}$ for $1 \mathrm{~min}$ to determine gene expression levels relative to those of a housekeeping gene, glyceraldehyde 3-phosphate dehydrogenase $(G A P D H)$. The primer sequences used for the genes analyzed are listed in Table I.

Animal studies. All animal experiments were approved by the Institutional Animal Care and Use Committee of the Asan Institute for Life Sciences, Korea (approval number 2015-02-170). For the mouse hepatic metastasis model, 8-week-old male Balb/c-nude mice (Central Animal Lab, Seoul, Korea) were anesthetized. Abdominal skin and wall tissues were incised, the median lobe was gently pulled up, and then the portal vein was exposed by carefully moving the intestine. An aliquot of $50 \mu \mathrm{l} \mathrm{M} 14$ cancer cells $\left(2 \times 10^{6}\right)$ was injected via the portal vein and then the puncture site was pressed with a sterilized cotton swab to ensure that the bleeding stopped. After injecting tumor cells, the intestine was repositioned and the abdominal wall was closed using a two-layer technique with nonabsorbable sutures. After 3 days, mice were divided into vehicletreated group $(n=11)$ and selumetinib-treated group $(n=12)$. Animals were treated daily (5 days per week) with vehicle ( $20 \%$ Captisol $^{\circledR}$, Ligand Pharmaceuticals, Inc., La Jolla, CA, USA) or $10 \mathrm{mg} / \mathrm{kg}$ selumetinib orally for 3 weeks. Selumetinib dose was that determined from our preliminary experiment and reference (16). At the end of experiments, livers were removed, weighed, and stored at $-80^{\circ} \mathrm{C}$ until usage

Statistical analysis. Data are presented as means \pm standard deviation. Significance was analyzed using Student's $t$-test with Prism 5 (Graph Pad Software Inc., San Diego, CA, USA) and $p$-values of 0.05 or less were considered as statistically significant.

\section{Results}

Selumetinib blocks constitutive ERK phosphorylation in human melanoma cell lines. In order to examine the inhibition efficacy of the MEK inhibitor selumetinib, we used human melanoma cell lines (M14 and UACC62). Cells were incubated with different concentrations of selumetinib for $24 \mathrm{~h}$. As shown in Figure 1, treatment of human melanoma cell lines with selumetinib significantly reduced phosphorylated ERK expression in a dose-dependent manner. Constitutive ERK phosphorylation was completely inhibited at a dose of $0.1 \mu \mathrm{M}$ selumetinib without notably affecting the total cellular level of ERK.

Selumetinib blocks cell-cycle progression and induces apoptosis in M14 cells. To determine the mechanism of actions of selumetinib, we analyzed the cell-cycle distribution and apoptosis in M14 cells. Cell-cycle analysis showed that M14 cells treated with $100 \mathrm{nM}$ of selumetinib exhibited a time-dependent accumulation of cells at the $\mathrm{G}_{0} / \mathrm{G}_{1}$ phase and fewer cells in $\mathrm{S}$ and $\mathrm{G}_{2}$ phase (Figure $2 \mathrm{~A}$ ). Moreover, the population of $\mathrm{G}_{0} / \mathrm{G}_{1}$ cells was increased after treatment with selumetinib for $48 \mathrm{~h}$ in a dose-dependent manner (Figure 2C). Potential effects on apoptosis of the MEK inhibitor were further evaluated by annexin $\mathrm{V}$ staining. After incubation with selumetinib for $72 \mathrm{~h}$, apoptotic cells (early and late apoptosis) increased in a dose- and timedependent manner (Figure 2B and D).

We further investigated the effects of selumetinib on multiple signaling pathways that regulate cell cycle- and apoptosis-related proteins. M14 and UACC62 cells were treated with 50,100 , or $200 \mathrm{nM}$ of selumetinib for $48 \mathrm{~h}$ and the expression levels of cell-cycle regulators were determined by western blot analysis (Figure 3). Consistent with the accumulation of cells in $G_{1}$ phase, the protein expression levels of c-MYC and cyclin $\mathrm{D}_{1}$ were significantly reduced in selumetinib-treated melanoma cells. Conversely, the cyclin-dependent kinase inhibitor $\mathrm{p} 27^{\mathrm{KIP} 1}$ accumulated after MEK blockade in a dose-dependent manner. Consistent with the annexin V staining pattern, PARP cleavage was induced in selumetinib-treated melanoma cells and the protein expression of survivin was reduced. Taken together, these results suggest that selumetinib induces the inhibition of cell-cycle progression and the activation of apoptosis in human melanoma cells. 

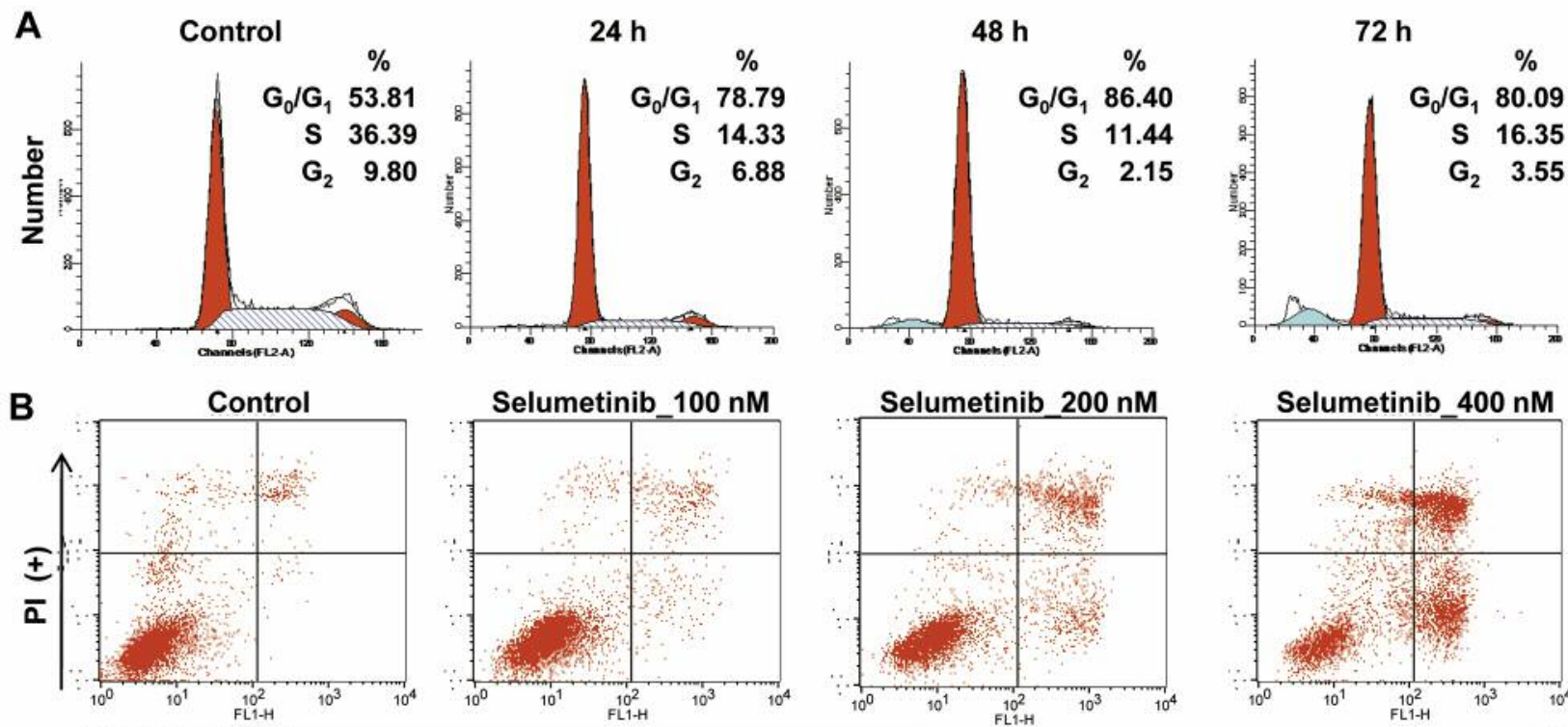

Selumetinib_100 $\mathrm{nM}$



Selumetinib_400 $\mathrm{nM}$

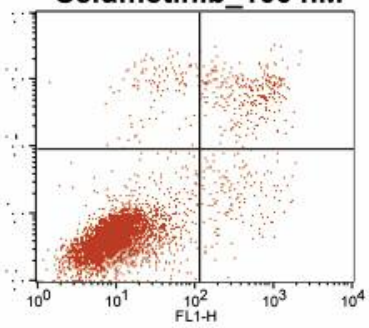

Annexin V(+)
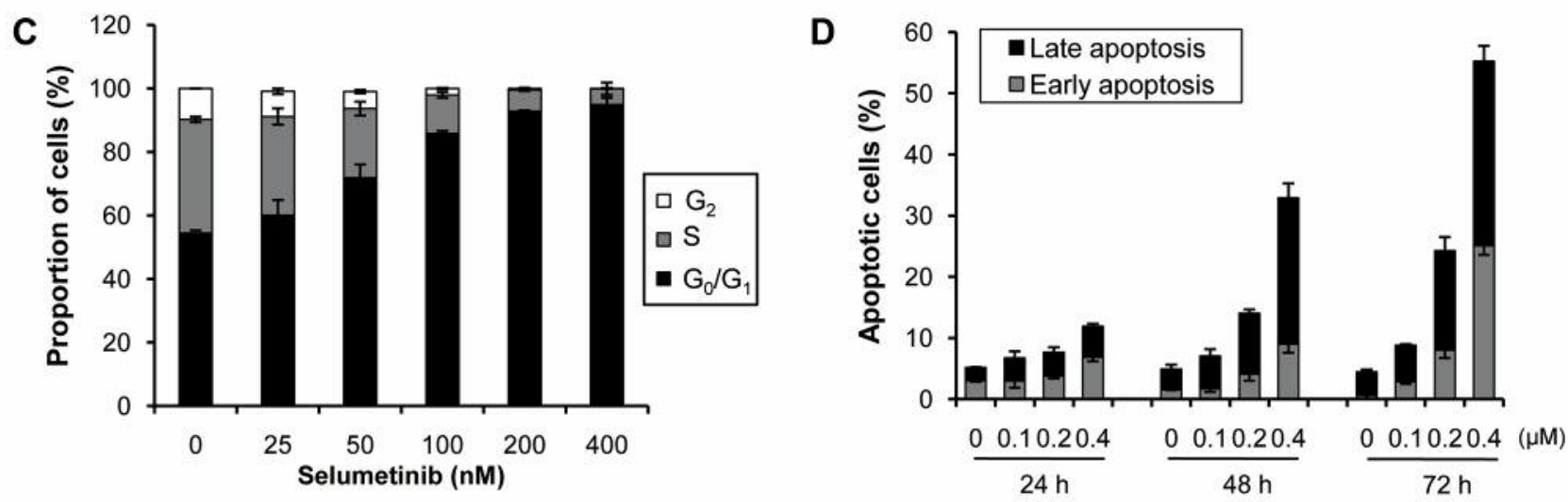

Figure 2. Blockade of cell-cycle progression and induction of apoptosis in M14 cells by selumetinib. A: Cell-cycle analysis revealed a proportional increase in sub- $G_{1}$ phase cells after treatment with $100 \mathrm{nM}$ of selumetinib for 24,48 and $72 \mathrm{~h}$. B: Induction of apoptosis in M14 cells by selumetinib treatment for $72 \mathrm{~h}$ based on annexin $V$ staining. $C$ : The population of $G_{0} / G_{1}$ cells was increased after treatment with selumetinib for $48 \mathrm{~h}$ in a dosedependent manner. D: Percentages of early and late apoptosis were calculated from flow cytometric analysis and selumetinib induced apoptosis in a time- and dose-dependent manner. Data are representative of two independent experiments.

Effects of selumetinib on transforming growth factor $\beta-1$ (TGF $\beta 1$ )-mediated transcription of metastasis-associated genes. TGF $\beta 1$ regulates the expression of various genes involved in biological phenomena, tissue remodeling, tumor initiation and progression. In addition, TGF $\beta 1$ can induce the epithelial-to-mesenchymal transition (EMT) and induces signaling pathways such as the SMAD and MAPK pathways. TGF $\beta 1$ is critical for EMT during tumor metastasis, and EMT results in the change in expression of many genes. Thus, to confirm the inhibition of cancer cell growth and EMT by selumetinib, we measured the expression of EMT-related genes in the TGF 31 -treated melanoma cell lines, M14 and UACC62, treated with selumetinib or not using reverse transcription-
PCR. Strikingly, selumetinib was able to inhibit the expression of NOTCH and FZD7 in both M14 and UACC62 cells (Figure 4). Serpin peptidase inhibitor clade E1 (SERPINEI) and matrix metallopeptidase 9 (MMP9) expression were activated by TGF $\beta 1$, whereas selumetinib suppressed the up-regulation of those genes. As a result, we propose that selumetinib reduces expression of EMT markers in TGF $\beta 1$-stimulated melanoma cells and is a potential therapeutic agent leading to growth inhibition and death of melanoma cells.

Selumetinib inhibits the progression of melanoma liver metastasis via suppression of metastasis-related genes. A melanoma liver metastasis model was established by 
A



Figure 3. Selumetinib modulates the expression of cell cycle- and apoptosis-related proteins. M14 (A) and UACC62 (B) cells were exposed to the indicated doses of selumetinib for $48 \mathrm{~h}$, then were subjected to western blot analysis. PARP: Poly ADP ribose polymerase.

injecting tumor cells into the portal vein of mice. Mice were randomly divided into vehicle- or selumetinib-treated groups 3 days after surgery. Administration of selumetinib for 3 weeks significantly blocked M14 cell liver metastasis compared with vehicle treatment. The numbers of tumor nodules of metastasized liver were reduced by selumetinib treatment (Figure 5A). To confirm this observation, mouse livers were removed and weighed (including both the liver and tumor nodules), and the liver weight per body weight was determined. The metastatic liver burden was $130.1 \pm 54.6 \mathrm{mg} / \mathrm{g}$ in vehicle-treated mice compared with $71.7 \pm 14.9 \mathrm{mg} / \mathrm{g}$ in selumetinib-treated mice, which exhibited significantly less tumor progression $(\mathrm{p}=0.0026$; Figure 5B). From this result, we suggest that selumetinib inhibits liver metastasis of melanoma cells in the mouse model of melanoma metastasis.

To strengthen this finding, we evaluated the expression levels of specific proteins and genes involved in tumor progression and metastasis. Firstly, in metastasized liver tissues, the protein expression levels of c-MYC were found to be elevated and this was suppressed by selumetinib (Figure 5C). Phosphorylated ERK was also detected in livers from vehicle-treated mice, whereas it was completely blocked by the administration of selumetinib, consistent with data from in vitro studies. Because metastasis was dramatically blocked in the selumetinib-treated group, we further evaluated the expression of metastasis-related genes in livers from normal control, vehicle-, and selumetinibtreated mice. In vehicle-treated metastasized liver tissues,
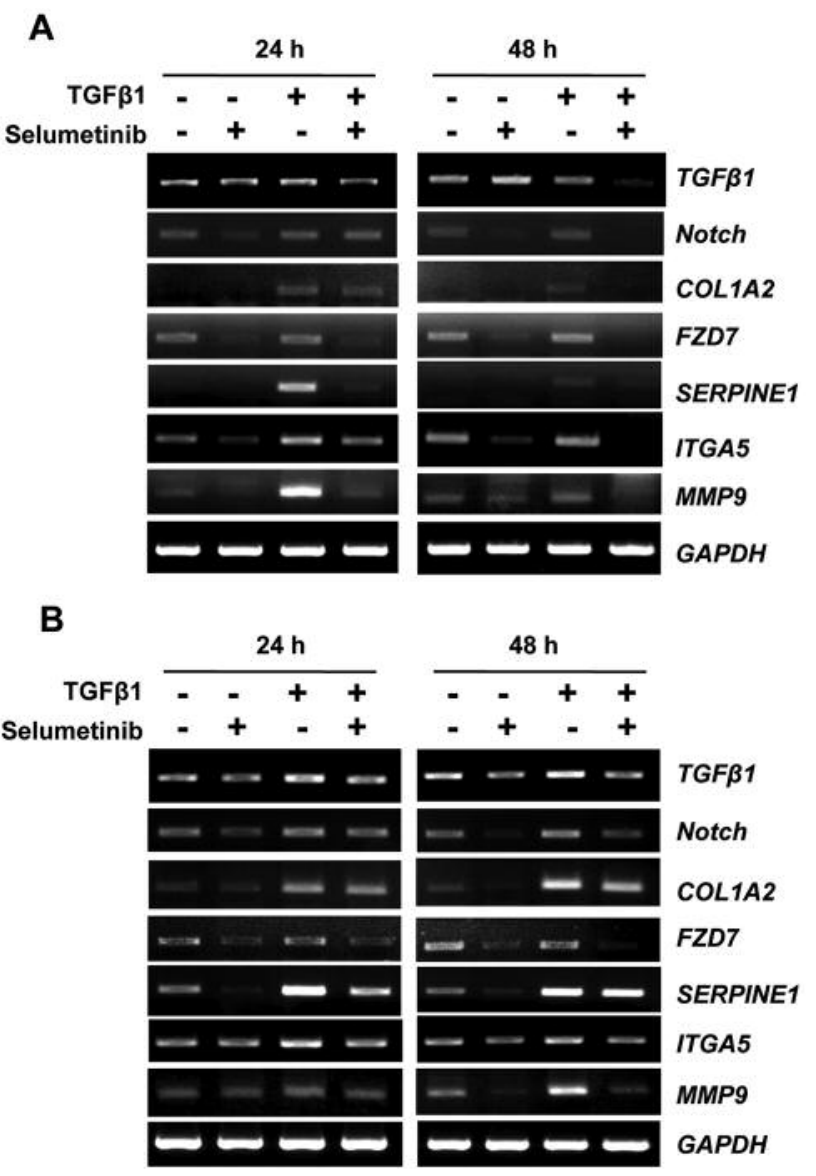

Figure 4. Selumetinib inhibits transforming growth factor $\beta$ (TGF $\beta$ )induced expression of epithelial-to-mesenchymal transition-related genes. M14 (A) and UACC62 (B) cells were treated with $200 \mathrm{nM}$ selumetinib with or without TGFB1 $(5 \mathrm{ng} / \mathrm{ml})$ for 24 or $48 \mathrm{~h}$. The expression levels of the indicated genes were measured by reverse transcription polymerase chain reaction. Primer sequences are listed in Table I. COL1A: Collagen type I alpha 2 chain, FZD7: frizzled-7, ITGA5: integrin alpha-5, JAG1: jagged 1, MMP9: matrix metallopeptidase 9, SERPINE1: serpin peptidase inhibitor clade E, TGF 1 1: transforming growth factor beta 1, ZEB1: zinc finger E-box-binding homeobox 1, GAPDH: glyceraldehyde 3-phosphate dehydrogenase.

levels of $T G F \beta 1$ expression were significantly elevated (Figure 5D). However, this change in expression was not detected in normal liver tissues and was expression decreased in the selumetinib-treated group. Because we used human TGF $\beta 1$ primers for RT-PCR, this finding can potentially explain why different expression levels were detected between normal and metastatic liver tissues. Thus, we identified that EMT-related genes were up-regulated in metastatic liver tissues. Interestingly, oral treatment with selumetinib blocked melanoma metastasis to the liver and inhibited the expression of EMT-/metastasis-target genes in this mouse model of melanoma metastasis. 
A

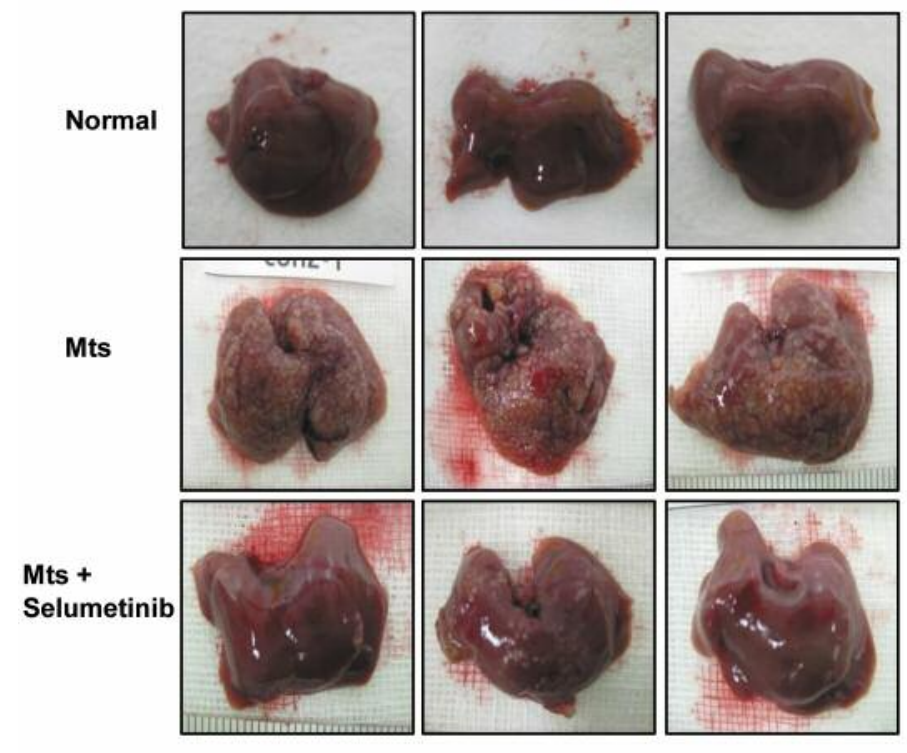

C

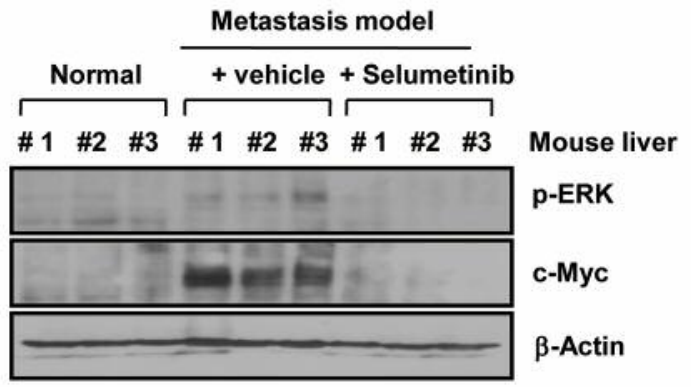

B
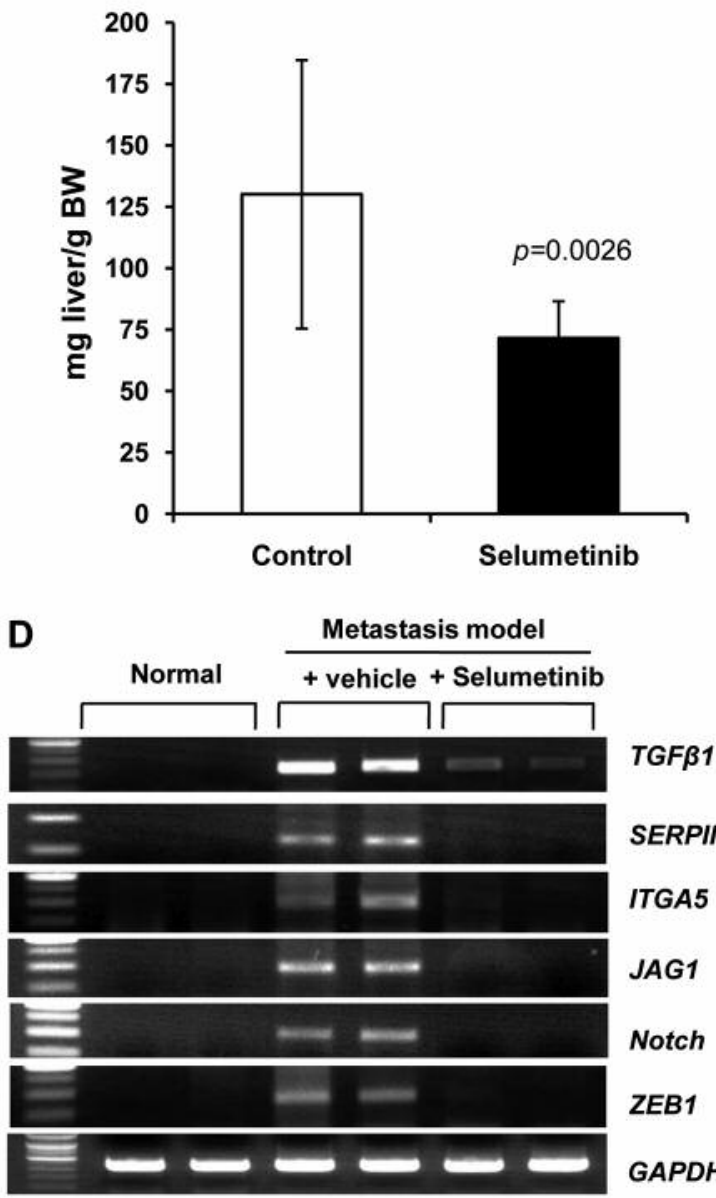

SERPINE1

ITGA5

JAG1

Notch

ZEB1

GAPDH

Figure 5. Selumetinib inhibits the progression of melanoma liver metastasis via suppression of metastasis-related genes. A: Representative images of metastases that developed in liver in mice. B: The average liver weight per body weight $(B W)$ of mice treated with vehicle- $(n=11)$ or selumetinib $(n=12)$. Data shown are the mean \pm standard deviation. C: Expression levels of phospho-extracellular signal-regulated kinase (p-ERK) and c-MYC increased in metastatic liver tissues; however, orally administered selumetinib blocked such changes in protein expression. D: Expression levels of metastasis-related genes were increased in liver metastases, as assessed using reverse transcription polymerase chain reaction. COL1A: Collagen type I alpha 2 chain, FZD7: frizzled-7, ITGA5: integrin alpha-5, JAG1: jagged 1, MMP9: matrix metallopeptidase 9, SERPINE1: serpin peptidase inhibitor clade E, TGF $\beta 1$ : transforming growth factor beta 1, ZEB1: zinc finger E-box-binding homeobox 1, GAPDH: glyceraldehyde 3-phosphate dehydrogenase.

\section{Discussion}

In our present study, we showed that a MEK inhibitor (selumetinib) prevents melanoma metastasis to the liver in a mouse model. Malignant melanoma exhibits strong resistance to traditional chemotherapeutic drugs and radiation treatment (17). MEK inhibition is an effective method for blocking the RAS/RAF/MEK/ERK signalling pathway in the treatment of melanoma (17). Previously, selumetinib was shown to block MEK activity and ERK phosphorylation in melanoma cell lines in vitro and in a mouse xenograft model in vivo $(18,19)$. We interpret those results as indicators that selumetinib has cytostatic rather than cytotoxic effects and they also suggest the potential clinical utility of a MEK inhibitor in preventing the early stages of metastasis. CI1040 , another type of MEK inhibitor, has also been shown to exhibit a cytostatic effect that can prevent lung metastasis in melanoma (20).

Metastasis is defined as the migration of cancer cells from the primary site to distant organs and represents the major cause of cancer-related deaths. To more fully elucidate the anti-metastatic effects of MEK inhibition, establishing an animal model is essential. Herein, we successfully established a mouse model of liver metastasis using a human melanoma cell line. Generally, hepatic metastasis models, which involve the injection of tumor cells into the liver 
parenchyma, spleen, or portal vein, have been widely used for studies of metastasis. Among these models, the intraportal vein injection model has been considered to be an ideal model for hepatic metastasis, but survival rates for this approach are often variable because of the difficulty in performing hemostasis. In the current study, to achieve a stable model of portal vein injection, we used adhesive glue (histo-bond) after the injection of tumor cells into the portal vein, which prevented excessive bleeding from the injection site. However, a limitation of this experimental metastasis model was that the metastasis that developed was not induced from a primary tumor, as shown in clinical cases. MEK inhibition can prevent liver metastasis by inhibiting the proliferation of M14 cells in the liver, and this finding is also supported by previous in vitro results. However, it remains unclear whether the MEK inhibitor blocked M14 cell proliferation in the blood stream, and then consequently reduced liver metastasis.

Many of the molecular changes associated with the transition of melanoma cells to the most advanced phases are unknown. The EMT of cancer cells is known to be a key event in the process of cancer metastasis. The RAS/RAF/MEK/ERK pathway is a major signaling pathway for EMT and metastasis (21-23). We also studied EMT-related genes, which might be inhibited by a MEK inhibitor. We found that MEK inhibition suppressed the expression of NOTCH, FZD7, SERPINE1, and MMP9. Cole et al. reported that MEK inhibitors suppressed the expression of metastasis-related genes in tumor cells (24). We propose that suppression of MMP expression and activity may represent an effective therapeutic approach for preventing cancer cell metastasis. The MMPs are a major class of molecules which modulate the extracellular matrix, which is a key step in the formation of metastasis. MMP activity has been considered to be an index of tumor invasion and metastatic potential $(25,26)$. In the present study, we observed that the suppression of MMP induced by MEK inhibition reduced liver metastasis. The MEK inhibitor suppressed MMP9 activity in vitro and liver metastasis in vivo. Expression of both MMP9 and SERPINE1 has been detected in more invasive and metastatic melanomas $(27,28)$.

In summary, mice with established liver metastases treated with a MEK inhibitor experienced significantly less tumor progression compared with mice that received vehicle alone. Our findings suggest that blockade of MEK signaling may prevent the progression of melanoma liver metastases and that MEK inhibitors maybe used in the treatment of metastatic cancer.

\section{Conflicts of Interest}

The Authors declare that they have no conflict of interest.

\section{Acknowledgements}

This study was supported by a National Research Foundation of Korea (NRF) grant funded by the Korean government (MEST; No. NRF-2012R1A2A2A01046062), and a grant from Asan Institute for Life Sciences (2014-308), Seoul, Korea.

\section{References}

1 Hau HM, Tautenhahn HM, Schoenberg MB, Atanasov G, Wiltberger G, Morgul MH, Uhlmann D, Seitz AT, Simon JC, Schmelzle $\mathrm{M}$ and Bartels $\mathrm{M}$ : Liver resection in multimodal concepts improves survival of metastatic melanoma: a singlecentre case-matched control study. Anticancer Res 34: 66336639, 2014.

2 Balch CM, Gershenwald JE, Soong SJ, Thompson JF, Atkins MB, Byrd DR, Buzaid AC, Cochran AJ, Coit DG, Ding S, Eggermont AM, Flaherty KT, Gimotty PA, Kirkwood JM, McMasters KM, Mihm MC Jr., Morton DL, Ross MI, Sober AJ and Sondak VK: Final version of 2009 AJCC Melanoma Staging and Classification. J Clin Oncol 27: 6199-6206, 2009.

3 Olofsson R, Cahlin C, All-Ericsson C, Hashimi F, Mattsson J, Rizell $\mathrm{M}$ and Lindner P: Isolated hepatic perfusion for ocular melanoma metastasis: registry data suggests a survival benefit. Ann Surg Oncol 21: 466-472, 2014.

4 Diener-West M, Reynolds SM, Agugliaro DJ, Caldwell R, Cumming K, Earle JD, Hawkins BS, Hayman JA, Jaiyesimi I, Jampol LM, Kirkwood JM, Koh WJ, Robertson DM, Shaw JM, Straatsma BR and Thoma J: Development of metastatic disease after enrollment in the COMS trials for treatment of choroidal melanoma: Collaborative Ocular Melanoma Study Group Report No. 26. Arch Ophthalmol 123: 1639-1643, 2005.

5 Mariani P, Piperno-Neumann S, Servois V, Berry MG, Dorval T, Plancher C, Couturier J, Levy-Gabriel C, Lumbroso-Le Rouic L, Desjardins L and Salmon RJ: Surgical management of liver metastases from uveal melanoma: 16 years' experience at the Institut Curie. Eur J Surg Oncol 35: 1192-1197, 2009.

6 Wellbrock C, Karasarides M and Marais R: The RAF proteins take centre stage. Nat Rev Mol Cell Biol 5: 875-885, 2004.

7 Kim DW and Patel SP: Profile of selumetinib and its potential in the treatment of melanoma. Onco Targets Ther 7: 1631-1639, 2014.

8 Patel SP and Kim KB: Selumetinib (AZD6244; ARRY-142886) in the treatment of metastatic melanoma. Expert Opin Investig Drugs 21: 531-539, 2012.

9 Karasarides M, Chiloeches A, Hayward R, Niculescu-Duvaz D, Scanlon I, Friedlos F, Ogilvie L, Hedley D, Martin J, Marshall $\mathrm{CJ}$, Springer CJ and Marais R: B-RAF is a therapeutic target in melanoma. Oncogene 23: 6292-6298, 2004.

10 Eisen T, Ahmad T, Flaherty KT, Gore M, Kaye S, Marais R, Gibbens I, Hackett S, James M, Schuchter LM, Nathanson KL, Xia C, Simantov R, Schwartz B, Poulin-Costello M, O’Dwyer PJ and Ratain MJ: Sorafenib in advanced melanoma: a Phase II randomised discontinuation trial analysis. Br J Cancer 95: 581586, 2006.

11 Alessi DR, Cuenda A, Cohen P, Dudley DT and Saltiel AR: PD 098059 is a specific inhibitor of the activation of mitogenactivated protein kinase kinase in vitro and in vivo. J Biol Chem 270: 27489-27494, 1995. 
12 Lorusso PM, Adjei AA, Varterasian M, Gadgeel S, Reid J, Mitchell DY, Hanson L, DeLuca P, Bruzek L, Piens J, Asbury P, Van Becelaere K, Herrera R, Sebolt-Leopold J and Meyer MB: Phase I and pharmacodynamic study of the oral MEK inhibitor CI-1040 in patients with advanced malignancies. J Clin Oncol 23: 5281-5293, 2005.

13 Adjei AA, Cohen RB, Franklin W, Morris C, Wilson D, Molina JR, Hanson LJ, Gore L, Chow L, Leong S, Maloney L, Gordon G, Simmons H, Marlow A, Litwiler K, Brown S, Poch G, Kane $\mathrm{K}$, Haney $\mathrm{J}$ and Eckhardt SG: Phase I pharmacokinetic and pharmacodynamic study of the oral, small-molecule mitogenactivated protein kinase kinase 1/2 inhibitor AZD6244 (ARRY142886) in patients with advanced cancers. J Clin Oncol 26: 2139-2146, 2008.

14 Infante JR, Fecher LA, Falchook GS, Nallapareddy S, Gordon MS, Becerra C, DeMarini DJ, Cox DS, Xu Y, Morris SR, Peddareddigari VG, Le NT, Hart L, Bendell JC, Eckhardt G, Kurzrock R, Flaherty K, Burris HA, 3rd and Messersmith WA: Safety, pharmacokinetic, pharmacodynamic, and efficacy data for the oral MEK inhibitor trametinib: a phase 1 dose-escalation trial. Lancet Oncol 13: 773-781, 2012.

15 Catalanotti F, Solit DB, Pulitzer MP, Berger MF, Scott SN, Iyriboz T, Lacouture ME, Panageas KS, Wolchok JD, Carvajal RD, Schwartz GK, Rosen N and Chapman PB: Phase II trial of MEK inhibitor selumetinib (AZD6244, ARRY-142886) in patients with $B R A F \mathrm{~V} 600 \mathrm{E} / \mathrm{K}$-mutated melanoma. Clin Cancer Res 19: 2257-2264, 2013.

16 Shannon AM, Telfer BA, Smith PD, Babur M, Logie A, Wilkinson RW, Debray C, Stratford IJ, Williams KJ and Wedge SR: The mitogen-activated protein/extracellular signal-regulated kinase kinase 1/2 inhibitor AZD6244 (ARRY-142886) enhances the radiation responsiveness of lung and colorectal tumor senografts. Clin Cancer Res 15: 6619-6629, 2009.

17 Cheng Y, Zhang G and Li G: Targeting MAPK pathway in melanoma therapy. Cancer Metastasis Rev 32: 567-584, 2013.

18 Haass NK, Sproesser K, Nguyen TK, Contractor R, Medina CA, Nathanson KL, Herlyn $M$ and Smalley KS: The mitogenactivated protein/extracellular signal-regulated kinase kinase inhibitor AZD6244 (ARRY-142886) induces growth arrest in melanoma cells and tumor regression when combined with docetaxel. Clin Cancer Res 14: 230-239, 2008.

19 Gopal YN, Deng W, Woodman SE, Komurov K, Ram P, Smith PD and Davies MA: Basal and treatment-induced activation of AKT mediates resistance to cell death by AZD6244 (ARRY142886) in $B R A F$-mutant human cutaneous melanoma cells. Cancer Res 70: 8736-8747, 2010.
20 Kramer BW, Gotz R and Rapp UR: Use of mitogenic cascade blockers for treatment of C-RAF-induced lung adenoma in vivo: CI-1040 strongly reduces growth and improves lung structure. BMC Cancer 4: 24, 2004.

21 Shirakihara T, Horiguchi K, Miyazawa K, Ehata S, Shibata T, Morita I, Miyazono K and Saitoh M: TGF-beta regulates isoform switching of FGF receptors and epithelial-mesenchymal transition. EMBO J 30: 783-795, 2011.

22 Halaban R, Zhang W, Bacchiocchi A, Cheng E, Parisi F, Ariyan S, Krauthammer M, McCusker JP, Kluger Y and Sznol M: PLX4032, a selective $B R A F(\mathrm{~V} 600 \mathrm{E})$ kinase inhibitor, activates the ERK pathway and enhances cell migration and proliferation of BRAF melanoma cells. Pigment Cell Melanoma Res 23: 190-200, 2010.

23 Nakabayashi H and Shimizu K: HA1077, a Rho kinase inhibitor, suppresses glioma-induced angiogenesis by targeting the RhoROCK and the mitogen-activated protein kinase kinase/ extracellular signal-regulated kinase (MEK/ERK) signal pathways. Cancer Sci 102: 393-399, 2011.

24 Cole GW Jr., Alleva AM, Zuo JT, Sehgal SS, Yeow WS, Schrump DS and Nguyen DM: Suppression of pro-metastasis phenotypes expression in malignant pleural mesothelioma by the PI3K inhibitor LY294002 or the MEK inhibitor UO126. Anticancer Res 26: 809-821, 2006.

25 Kawata R, Shimada T, Maruyama S, Hisa Y, Takenaka H and Murakami Y: Enhanced production of matrix metalloproteinase2 in human head and neck carcinomas is correlated with lymph node metastasis. Acta Otolaryngol 122: 101-106, 2002.

26 Himelstein BP, Asada N, Carlton MR and Collins MH: Matrix metalloproteinase-9 (MMP9) expression in childhood osseous osteosarcoma. Med Pediatr Oncol 31: 471-474, 1998.

27 Klein RM, Bernstein D, Higgins SP, Higgins CE and Higgins PJ: SERPINE1 expression discriminates site-specific metastasis in human melanoma. Exp Dermatol 21: 551-554, 2012.

28 Hofmann UB, Westphal JR, Van Muijen GN and Ruiter DJ: Matrix metalloproteinases in human melanoma. J Invest Dermatol 115: 337-344, 2000.
Received November 22, 2016

Revised January 6, 2017

Accepted January 19, 2017 